\title{
Photo-Induced Spin-State Conversion in Solvated Transition Metal Complexes Probed via Time-Resolved Soft X-ray Spectroscopy
}

\author{
Nils Huse, ${ }^{1}$ Tae Kyu Kim, ${ }^{2}$ Lindsey Jamula, ${ }^{3}$ James K. McCusker, ${ }^{3}$ \\ Frank M. F. de Groot, ${ }^{4}$ and Robert W. Schoenlein ${ }^{1,5}$ \\ ${ }^{1}$ Ultrafast X-ray Science Lab, Chemical Sciences Division, Lawrence Berkeley National \\ Laboratory, Berkeley, CA 94720, USA, ${ }^{2}$ Department of Chemistry, Pusan National University, \\ Geumjeong-gu, Busan 609-735, Korea, ${ }^{3}$ Department of Chemistry, Michigan State University, \\ East Lansing, MI 48824, USA, ${ }^{4}$ Department of Chemistry, Utrecht University, \\ 3584 CA Utrecht, Netherlands, ${ }^{5}$ Material Sciences Division, Lawrence Berkeley National \\ Laboratory, Berkeley, CA 94720, USA \\ DOI: 10.1021/ja101381a, E-mail: nhuse@lbl.gov
}

\begin{abstract}
Solution-phase photo-induced low-spin to high-spin conversion in the Fe" polypyridyl complex $\left[\mathrm{Fe}\left(\operatorname{tren}(\mathrm{py})_{3}\right)\right]^{2+}$ (where tren $(\mathrm{py})_{3}$ is tris(2-pyridylmethylimino-ethyl)amine) has been studied via picosecond soft $x$-ray spectroscopy. Following ${ }^{1} \mathrm{~A}_{1} \rightarrow{ }^{1} \mathrm{MLCT}$ (metal-to-ligand charge transfer) excitation at $560 \mathrm{~nm}$, changes in the iron $L_{2}$ and $L_{3}$ edges were observed concomitant with formation of the transient high-spin ${ }^{5} T_{2}$ state. Charge-transfer multiplet calculations coupled with data acquired on low-spin and high-spin model complexes revealed a reduction in ligand field splitting of $\sim 1 \mathrm{eV}$ in the high-spin state relative to the singlet ground state. A significant reduction in orbital overlap between the central Fe-3d and the ligand $\mathrm{N}-2 p$ orbitals was directly observed, consistent with the expected ca. $0.2 \AA$ increase in Fe-N bond length upon formation of the high-spin state. The overall occupancy of the Fe $3 d$-orbitals remains constant upon spin-crossover, suggesting that the reduction in $\sigma$-donation is compensated by significant attenuation of $\pi$-back-bonding in the metal-ligand interactions. These results demonstrate the feasibility and unique potential of time-resolved soft $x$-ray absorption spectroscopy to study ultrafast reactions in the liquid phase by directly probing the valence orbitals of first-row metals as well as lighter elements during the course of photochemical transformations.
\end{abstract}

\section{Introduction}

Observing the evolution of valence-charge distributions during chemical reactions in real time is of fundamental importance for understanding the rearrangement of chemical bonds, their cooperative relationship with atomic position, and the creation of new chemical species. Time-resolved spectroscopy in the visible and infrared regions has been widely applied for elucidating chemical dynamics at the molecular level by probing vibrational and electronic degrees of freedom. ${ }^{1-6}$ Such studies have provided important information concerning excited-state dynamics with implications in areas ranging from chemistry and physics to biology and materials science. Nevertheless, there are often significant limitations of electronic and vibrational spectroscopies: the sometimes broad, nondescript nature of electronic spectra and the inherent vibrational complexity of large molecular frameworks can make interpretation of time-resolved optical and infrared and/or Raman data challenging. This is a common situation in the spectroscopy of transition metal-containing systems, ${ }^{7}$ wherein the density of both electronic and vibrational states can render mechanistic questions concerning photo-induced dynamics difficult to resolve.

The emerging technique of ultrafast $\mathrm{x}$-ray spectroscopy ${ }^{8-}$ 13 provides important new insights into molecular dynamics because $\mathrm{x}$-ray core-level transitions (e.g. $2 p_{1 / 2} \rightarrow$ LUMO) are element specific, symmetry and spin state selective, and effective for probing lighter elements $(\mathrm{C}, \mathrm{N}, \mathrm{O}$ etc.) that are biologically relevant. ${ }^{14}$ In contrast to visible spectroscopy, in which the mixed orbital character of the probed HOMO and LUMO states often precludes a detailed understanding of the observed spectra, x-ray spectroscopy exploits core-level tran- sitions from states of well-defined symmetry to extract more detailed information on the final states and hence a wealth of information about the systems under study. Accordingly, the $\mathrm{x}$-ray near-edge spectral region (XANES) provides a quantitative fingerprint of the local chemical bonding geometry (orbital hybridization etc.) in the vicinity of the atom(s) of interest. This is particularly powerful for understanding molecular dynamics in solution, where much important chemistry occurs, and where the solvent environment substantially influences reaction dynamics.

One of the unique advantages of L-edge spectroscopy for the study of transition metal compounds is the fact that it provides a direct probe of valence orbitals. In the case of firstrow metal complexes, the L-edge can provide critical information about the $3 d$-orbitals: since these orbitals largely define the chemistry of this class of molecules, such measurements can yield fundamental insights into bonding in these compounds that compliments information obtained from the other forms of spectroscopy mentioned above. In a broader context, the prevalence of transition metals in biological systems ${ }^{15}$ underscores the importance for many research areas to understand the evolution of electronic structure associated with chemical reaction dynamics of such systems.

Herein we report the first picosecond soft x-ray spectroscopy of electronic dynamics in solvated transition-metal complexes. Specifically, we have acquired time-resolved Ledge XANES spectra of an $\mathrm{Fe}^{\mathrm{II}}$ complex following photoinduced charge-transfer in order to understand the evolution of the valence orbital structure subsequent to electronic excitation. The molecule that is the subject of the present study is $\left[\mathrm{Fe}\left(\operatorname{tren}(\mathrm{py})_{3}\right)\right]^{2+}$ (Figure 1). This compound is part of a larger class of inorganic compounds referred to as "spin-crossover" 

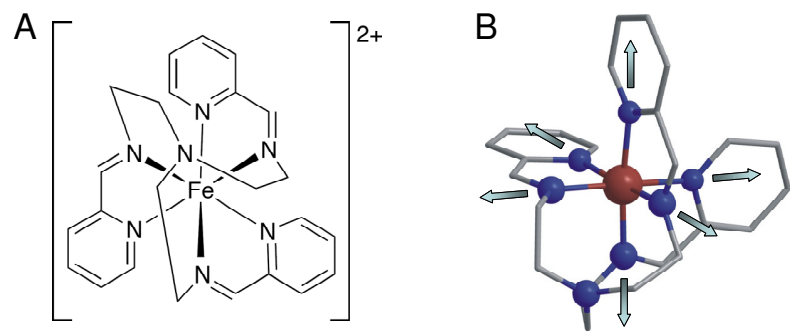

Figure 1. (A) Schematic drawing of the molecular structure of $\left[\mathrm{Fe}\left(\operatorname{tren}(\mathrm{py})_{3}\right)\right]^{2+}$. (B) Photoinduced metal-to-ligand charge transfer of $[\mathrm{Fe}(\operatorname{tren}(\mathrm{py}) 3)] 2+$ results in ultrafast structural changes. Arrows depict the ligand cage dilation as reported by Khalil and co-workers. ${ }^{33}$

compounds for their propensity to undergo interconversion between a low-spin and a high-spin configuration $(\mathrm{S}=0$ and $\mathrm{S}=2$ in the case of $\mathrm{Fe}^{\mathrm{II}}$ ) given an appropriate external perturbation (e.g. temperature or pressure). ${ }^{16}$ The transformation can also be effected upon photoexcitation as demonstrated by the so-called LIESST effect (Light-Induced Excited SpinState Trapping) first reported by Decurtins and co-wrokers. ${ }^{17}$ Interest in these widely studied compounds stems from the profound difference in optical, magnetic, and geometric properties endemic to the two accessible spin states, suggesting possible applications in magneto-optics among others. ${ }^{18,19}$ Of fundamental scientific interest is the complex coupling of electronic, nuclear and magnetic (spin \& orbital) degrees of freedom. This gives rise to coupled electronic and structural dynamics that are ideally suited for study by x-ray techniques. Our data reveal new insights into the nature of changes in metal-ligand interactions concomitant with the transition between the low-spin and high-spin states of the compound, thereby establishing the unique power of this form of spectroscopy to probe important aspects of photoinduced dynamics.

\section{Experimental Section}

X-Ray Parameters. Transient $\mathrm{x}$-ray absorption measurements were performed at the ultrafast x-ray facility of the Advanced Light Source ${ }^{20,21}$ capable of producing femtosecond X-rays between $200 \mathrm{eV}$ and $10 \mathrm{keV}$. A schematic drawing of the experiment is depicted in Figure 2. The facility incorporates a high-repetition-rate Ti:sapphire laser system that can produce frequency-converted excitation pulses and, for the soft $\mathrm{x}$-ray range, employs an undulator-based beamline, equipped with an x-ray chopper and a varied line-space grating monochromator, tunable from $200 \mathrm{eV}$ to $2000 \mathrm{eV}$.

The filling pattern of the Advanced Light Source synchrotron consists of 275 electron bunches spaced 2 ns apart. One so-called "camshaft" bunch (10 mA max. current, $70 \mathrm{ps}$ FWHM bunch duration) is isolated in a 100-ns wide gap as depicted on the left of Figure 2 to allow for gated detection of the X-rays produced by the camshaft bunch during passage through the magnetic undulator structure. ${ }^{23}$ Differential x-ray absorption spectra at the Fe L-edges were recorded from 700 $\mathrm{eV}$ to $730 \mathrm{eV}$ with an energy resolution of $0.5 \mathrm{eV}$. The X-rays were focused to $50 \mu \mathrm{m}$ at an attenuated flux of $10^{3}$ photons/pulse/ $0.1 \%$ bandwidth at the sample to prevent sample damage.

Laser Parameters. The 70 fs excitation pulses at 560 $\mathrm{nm}$ were derived from an optical parametric amplifier (Light Conversion TOPAS-C), and synchronized to the x-ray pulses with a precision of better than 5 picoseconds by locking the $62.5 \mathrm{MHz}$ repetition rate of the cavity-length-stabilized oscillator of the laser system to the $500 \mathrm{MHz}$ of the synchrotron's radio frequency cavity. The spectrum of the pump pulses was measured with a commercial spectrometer (Ocean Optics RedTide). The visible pump pulses were focused to $150 \mu \mathrm{m}$ at the sample $\left(4 \mu \mathrm{J} /\right.$ pulse, incident at $\sim 10^{\circ}$ with respect to the $\mathrm{X}$-rays) and detected outside the vacuum chamber with a silicon diode for alignment purposes.

Sample Preparation. A microcrystalline sample of $\left[\mathrm{Fe}\left(\operatorname{tren}(\mathrm{py})_{3}\right)\right]\left(\mathrm{PF}_{6}\right)_{2}$ was prepared following a previously published procedure. ${ }^{24}$ Purity of the sample was verified by elemental analysis and electrospray mass spectroscopy using the analytical facilities of Michigan State University. Solutions of $\left[\mathrm{Fe}\left(\operatorname{tren}(\mathrm{py})_{3}\right)\right]\left(\mathrm{PF}_{6}\right)_{2}$ in acetonitrile were prepared at $100 \mathrm{mM}$ concentration.

L-edges of the important first-row transition metals lie in the soft $\mathrm{x}$-ray region below $1 \mathrm{keV}$. Due to large absorption cross sections in this spectral region, transmission spectroscopy in liquid-phase requires sample films of a few micrometers in thickness that remain stable in a vacuum environment under typical photo-excitation conditions. ${ }^{25-28}$ The liquid film sample of $\left[\mathrm{Fe}\left(\operatorname{tren}(\mathrm{py})_{3}\right)\right]\left(\mathrm{PF}_{6}\right)_{2}$ was enclosed in a $0.6 \mathrm{~mm}$ wide channel between two $100 \mathrm{~nm}$-thick silicon nitride membranes held by silicon substrates which were separated by a 2 micron layer of photoresist. The latter covered almost all of the substrate material except for the aforementioned channel that traverses the substrate and the silicon nitride window. The entire sample cell was housed in an o-ring sealed metal enclosure and mounted in a vacuum chamber that was filled to 200 Torr helium pressure to offset the vapor pressure of the liquid. A third $100 \mathrm{~nm}$-thick silicon nitride membrane isolated the sample chamber from the ultrahigh vacuum of the soft $\mathrm{x}$ ray beamline. To reach the desired sample thickness of $2 \mu \mathrm{m}$, the interior cell pressure was balanced against the pressure of the sample chamber through a small tube that led from the inside of the metal enclosure out of the vacuum chamber to a pressure regulating setup. With this approach, the thickness of the liquid film can be controlled with sub-200 nm accuracy $^{29,30}$ as measured by transmission interferometry.

Pump-Probe Measurements. Spatial overlap of visible pump and x-ray probe pulses was ensured by alignment
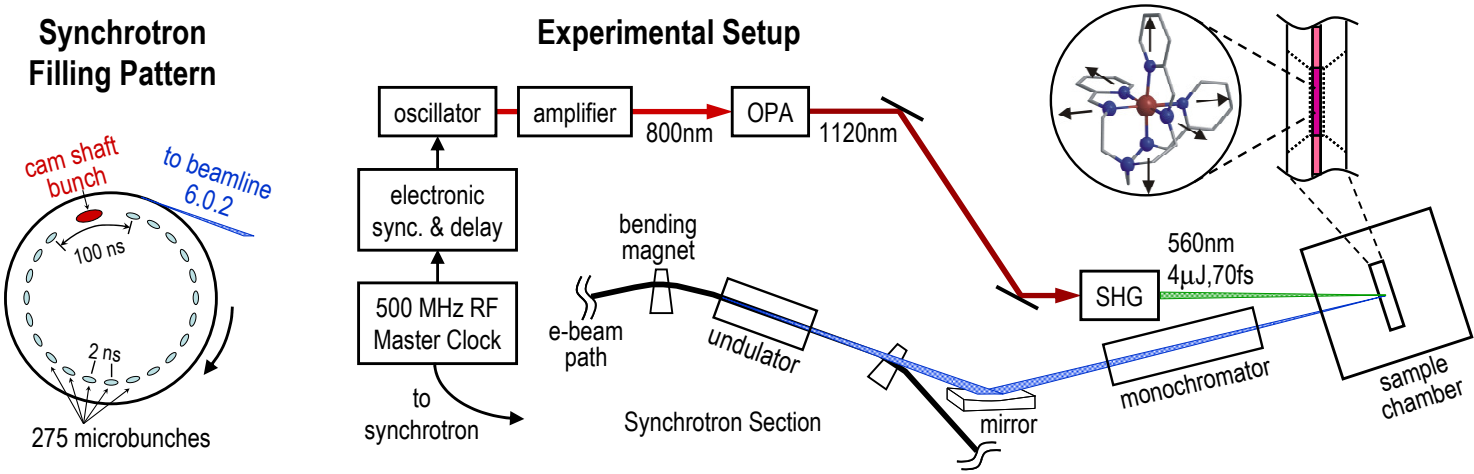

Figure 2. Schematic drawing of the electron bunch filling pattern (left) and the picosecond soft X-ray experiment (right) of the Advanced Light Source. A femtosecond Ti:sapphire laser system supplies 800-nm laser pulses synchronous with the X-ray pulses from the synchrotron. The initial laser pulses are frequencyconverted to $1120 \mathrm{~nm}$ pulses via an optical parametric amplifier (OPA) with subsequent second harmonic generation (SHG) in a $\beta$-barium borate crystal (BBO). The resulting 560-nm pulses excite the ${ }^{1} \mathrm{~A}_{1} \rightarrow{ }^{1} \mathrm{MLCT}$ transition of $\left[\mathrm{Fe}\left(\operatorname{tren}(\mathrm{py})_{3}\right)\right]^{2+}$ in the visible pump/X-ray probe experiment. 
Scheme 1. Schematic of Relevant Orbitals and Energetics Associated with Time-Resolved Soft X-ray Absorption Measurements ${ }^{a}$

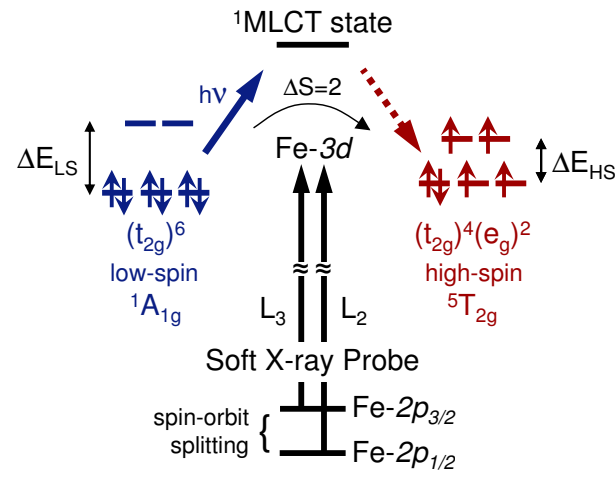

${ }^{a}$ The scheme illustrates how the photo-initiated intersystem crossing is probed via Fe $2 p \rightarrow 3 d$ dipole transitions. The ligand field surrounding the central metal in $\left[\mathrm{Fe}\left(\operatorname{tren}(\mathrm{py})_{3}\right)\right]^{2+}$ imposes an energy splitting, $\Delta E$, of $3 \mathrm{~d}$ orbitals into $\mathrm{t}_{2 \mathrm{~g}}$ and $\mathrm{e}_{\mathrm{g}}$ orbitals.

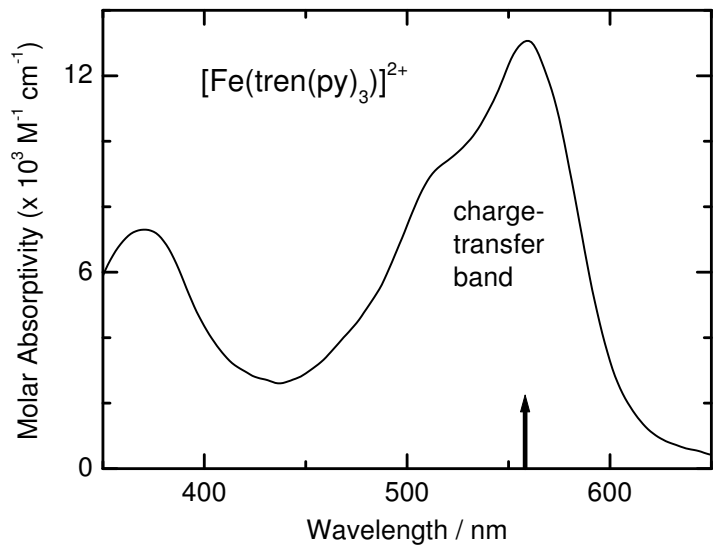

Figure 3. Electronic absorption spectrum of $\left[\mathrm{Fe}\left(\operatorname{tren}(\mathrm{py})_{3}\right)\right]\left(\mathrm{PF}_{6}\right)_{2}$ in $\mathrm{CH}_{3} \mathrm{CN}$ solution. The black arrow indicates the excitation wavelength in the ${ }^{1} \mathrm{~A}_{1} \rightarrow$ ${ }^{1}$ MLCT absorption envelope used in the present study.

through a pinhole placed in the sample plane. Temporal overlap of $<20$ ps accuracy was determined by measuring the response of a fast silicon avalanche photodiode with a $\mathrm{GHz}$ oscilloscope (Tektronix 5104B). For delay scans at fixed xray energy, the time delay between pump and probe pulses was varied electronically. The sample was excited at a repetition rate of $1 \mathrm{kHz}$ while the $\mathrm{x}$-ray pulses that probe the sample transmission were recorded at $2 \mathrm{kHz}$ thereby creating interleaved data of ground-state and photo-excited sample $\left(\mathrm{I}_{0}\right.$ and $I(t)$, respectively, where $t$ is the time delay between the pump and the probe pulses). The total integration time per data point was typically $20 \mathrm{~s}$.

Multiplet Calculations. The experimental L-edge spectra were modelled with the charge transfer multiplet (CTM) approach. The CTM approach is based on atomic theory, with the addition of crystal field interactions, and the CTM model of Thole and co-workers. ${ }^{31,32}$ The CTM many-body Hamiltonian includes the on-site correlation energy $U$ of the Fe-3d level, the ligand-to-metal charge transfer energy $\mathrm{D}$, the metalto-ligand charge transfer energy $\mathrm{D}^{\prime}$, the $3 d 3 d$ electronelectron interaction within the $3 d$ shell and the $2 p 3 d$ multiplet interaction between the $2 p$ and $3 d$ shells, as well as the spinorbit coupling in each subshell. The atomic values have been used for the $2 p 3 d$ and $3 d 3 d$ interactions and for the $2 p$ and $3 d$ spin-orbit coupling. The Fe environment was simulated using a crystal field with $O_{h}$ - symmetry defined by the crystal-field splitting parameter 10Dq. Covalent mixing between the metal $3 d$-orbitals with the ligand valence $2 p$-orbitals was taken into account by charge-transfer between the $3 d^{6}, 3 d^{5} L$, and $3 d^{7} \underline{L}$ configurations (the latter two describing $\pi$-back-bonding and $\sigma$-donation, respectively).

\section{Results and Discussion}

Before discussing our experimental findings, we introduce important details of our model compound. The low-spin $\left[\mathrm{Fe}\left(\operatorname{tren}(\mathrm{py})_{3}\right)\right]^{2+}$ complex has six nitrogen atoms in the primary coordination sphere interacting with the central iron atom. The influence of the tren(py $)_{3}$ ligand can be described to first approximation as presenting a ligand field of roughly octahedral symmetry $\left(O_{h}\right)$ that partially lifts the Fe-3d orbital degeneracy, resulting in a (nominally) three-fold degenerate $t_{2 g}$ set and a two-fold degenerate $e_{g}$ set (Scheme 1). The visible absorption spectrum of $\left[\mathrm{Fe}\left(\operatorname{tren}(\mathrm{py})_{3}\right)\right]^{2+}$ in $\mathrm{CH}_{3} \mathrm{CN}$ solution is plotted in Figure 3. The intense feature having $\lambda_{\max }=$ $560 \mathrm{~nm}$ is due to a spin-allowed metal-to-ligand chargetransfer (MLCT) transition (i.e., ${ }^{1} \mathrm{~A}_{1} \rightarrow{ }^{1} \mathrm{MLCT}$ ); in terms of an orbital description, this absorption corresponds roughly to a $\left(t_{2 g}\right)^{6}\left(\pi^{*}\right)^{0} \rightarrow\left(t_{2 g}\right)^{5}\left(\pi^{*}\right)^{1}$ transition, where the $\pi^{*}$ orbital is associated with the pyridyl rings of the tren $(\mathrm{py})_{3}$ ligand.

The excited-state dynamics of $\left[\mathrm{Fe}\left(\operatorname{tren}\left(\mathrm{py}_{3}\right)\right]^{2+}\right.$ have been the subject of several previous studies. ${ }^{24,33,34}$ In 2000 , Monat and McCusker used a combination of spectroelectrochemistry and time-resolved electronic absorption spectroscopy to characterize a sub-100 fs process by which the initially formed ${ }^{1}$ MLCT state converts to the ligand-field manifold; subsequent kinetics on the time scale of several picoseconds were ascribed to vibrational cooling in the ${ }^{5} \mathrm{~T}_{2}$ state based on an equivalence between the transient spectrum at $\Delta \mathrm{t}>1 \mathrm{ps}$ and the expected high-spin/low-spin differential absorption profile of the compound. ${ }^{24}$ These efforts were extended into the ultraviolet region by Smeigh and co-workers which served to define the time scale for formation of the known ground-state/excited-state isosbestic point in the compound's electronic absorption spectrum. ${ }^{34}$ Significantly, this report also detailed the use of femtosecond stimulated Raman scattering to examine changes in the vibrational structure of the compound: the confluence of data established a time constant of $\tau=190 \pm 50$ fs for the formation of the ${ }^{5} \mathrm{~T}_{2}$ state in solution. Studies by Chergui and co-workers on $\left[\mathrm{Fe}(\mathrm{bpy})_{3}\right]^{2+}$ (where bpy is 2,2'-bipyridine) yielded similar conclusions in terms of extremely rapid formation of the high-spin excited state, ${ }^{35,36}$ suggesting that sub-picosecond, formally $\Delta S=2$ spin-state conversion might be a common feature following charge-transfer excitation of low-spin iron polypyridyl complexes.

Transient hard X-ray spectroscopy of the extended X-ray absorption fine structure (EXAFS) at the Fe K-edge of $\left[\mathrm{Fe}\left(\operatorname{tren}(\mathrm{py})_{3}\right)\right]^{2+}$ and similar systems ${ }^{33,38,39}$ has revealed detailed structural aspects associated with the MLCT transition, indicating that the electronic changes that follow optical excitation are concurrent with a dilation of the $\mathrm{Fe}-\mathrm{N}$ bonds of ca. $0.2 \AA$. This remarkably fast transformation should therefore be coupled to changes in Fe- $3 d / \mathrm{N}-2 p$ hybridization due to a weakening of the metal-ligand bonds as well as associated changes in the ligand-field splitting of the Fe- $3 d$ orbitals. However, transient K-edge spectroscopy near the X-ray absorption edge of first-row transition metals $(1 s \rightarrow 4 p$ XANES) reveals much less information on electronic changes than transient L-edge spectroscopy due to the increased lifetimebroadening of spectral features at the metal K-edge and small signals from dipole-forbidden transitions between the initial states and the valence states of interest, e.g. Fe- $1 s$ and Fe-3d. Time-resolved spectroscopy of transition-metal L-edges directly probes changes in ligand-field split metal- $d$ orbitals and spin states via selective excitations of the spin-orbit-split metal- $2 p_{1 / 2}$ and $2 p_{3 / 2}$ electrons to vacancies in the Fe- $3 d$ valence orbitals. Generally, this observation method of the valence electronic states has the potential of delivering detailed 


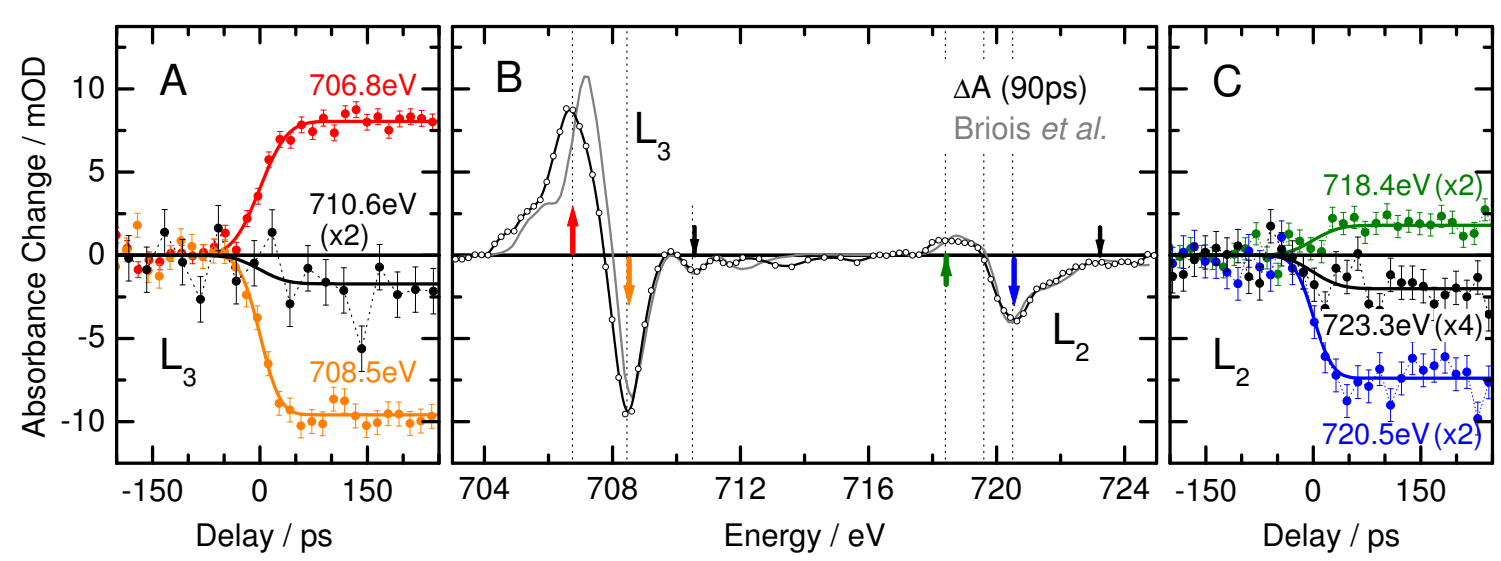

Figure 4. Absorbance changes at the Fe L-edges derived from transmitted X-ray intensities prior and subsequent to excitation of $\left[\mathrm{Fe}\left(\operatorname{tren}(\mathrm{py})_{3}\right)\right]^{2+}$ at $560 \mathrm{~nm}$. $(\mathrm{B})$ Differential absorption spectrum 90 ps after photoexcitation (open circles with spline fit as black line). For comparison, we plotted the difference of two static spectra (gray line) of a similar crystalline spin-crossover compound FeII(phen $)_{2}(\mathrm{NCS})_{2}$ (where phen ) 1,10-phenanthroline), which were recorded above and below the critical temperature $T_{\mathrm{c}}$ that separates the high-spin phase $\left(>T_{\mathrm{c}}\right)$ from the low-spin phase $\left(<T_{\mathrm{c}}\right){ }^{54}(\mathrm{~A}, \mathrm{C})$ Absorbance changes as a function of time delay between visible pump and X-ray probe pulses at the $\mathrm{L}_{3}$-edge and $\mathrm{L}_{2}$-edge. Solid lines are fits to the data based on a model of instantaneous absorption changes convolved with a 70 ps pulse and negligible relaxation within the delay window.

information about transition states in chemical reactions that has so far been inaccessible.

Transient L-edge Spectroscopy. X-ray absorption data acquired following ${ }^{\mathrm{P}} \mathrm{A}_{1} \rightarrow{ }^{1} \mathrm{MLCT}$ excitation of [Fe(tren$\left.\left.(\mathrm{py})_{3}\right)\right]^{2+}$ in $\mathrm{CH}_{3} \mathrm{CN}$ solution are shown in Figure 4: this is to our knowledge the first time-resolved absorption spectrum of solvated molecules ever recorded in the soft x-ray region. The differential absorption spectrum at 90 ps delay is shown in the center panel of Figure 4 . At the $\mathrm{Fe}_{3}$-edge, the differential spectrum displays an asymmetric dispersive shape that is indicative of an absorption shift to lower energies accompanied by some reshaping of the $\mathrm{L}_{3}$-edge. At the $\mathrm{L}_{2}$-edge, a pronounced decrease in absorption at $\sim 720 \mathrm{eV}$ is accompanied by a distinct but smaller absorption increase at lower energies. These absorption changes signal a significant reshaping of the $\mathrm{L}_{2}$-edge that dominates the accompanying absorption shift to lower energies. We verified that the shape and amplitude of the entire differential absorption spectrum is constant for delays up to $5 \mathrm{~ns}{ }^{40}$

The integral over all recorded energies is essentially zero while the integrated absorption change is positive at the $\mathrm{L}_{3}-$ edge and negative at the $\mathrm{L}_{2}$-edge. This implies that the absorption is conserved upon photo-initiated spin crossover but weight is shifted from the $\mathrm{L}_{2}$-edge to the $\mathrm{L}_{3}$-edge. To quantify this spectral change, we use the branching ratio $\mathrm{B}$ between the absorption spectral weight at the $\mathrm{L}_{3}$ and $\mathrm{L}_{2}$ edges defined as $\mathrm{B}=\mathrm{L}_{3} /\left(\mathrm{L}_{3}+\mathrm{L}_{2}\right)$. The increase of the $\mathrm{L}_{3}$ edge absorption at the expense of the $\mathrm{L}_{2}$ edge absorption, and subsequently the increase of the branching ratio, directly indicates the increase of the spin state populations and reflects the underlying spinstate selectivity of the Fe- $2 p$ transitions imposed by the dipole selection rules. ${ }^{41-43}$ In crystal field multiplet theory, the branching ratio is predicted to be $\mathrm{B}_{\mathrm{HS}} \approx 0.85$ for high-spin $\mathrm{Fe}^{\mathrm{II}}$ systems and $\mathrm{B}_{\mathrm{LS}} \approx 0.6$ for low-spin $\mathrm{Fe}^{\mathrm{II}}$ systems. ${ }^{4,45} \mathrm{We}$ find branching ratios of $\mathrm{B}_{\mathrm{HS}}=0.80$ and $\mathrm{B}_{\mathrm{LS}}=0.65$ which is close to the above values. Metal-ligand interactions (electron delocalization) and deviations from octahedral symmetry influence the branching ratio as does the choice of integral boundaries when calculating the spectral weight of both edges. An important consequence of constant absorption crosssection upon spin-crossover is the implication that the effective occupancy of the $\mathrm{Fe}-3 d$ orbitals does not change, i.e. there is no discernable change in the overall degree of valence charge distribution upon low-spin to high-spin conversion.

We also recorded the absorption changes as function of pump-probe delay at several probe energies as shown in panels $\mathrm{A}$ and $\mathrm{C}$ of Figure 4. The transients in these panels follow the absorption changes at the $\mathrm{L}_{3}$-edge and $\mathrm{L}_{2}$-edge, respectively. The absorption decreases around $708.5 \mathrm{eV}$ and 720.5 $\mathrm{eV}$ and increases around $707 \mathrm{eV}$ and $719 \mathrm{eV}$, complementing the observed changes in the spectral domain (Figure 4B). The transients also serve to differentiate the spectral baseline and signals from noise. For example, the weak signals in the differential spectrum of Figure 4B (black arrows in the center panel) around $710.5 \mathrm{eV}$ and beyond $722 \mathrm{eV}$ are reproduced by the transients at these probe energies (black traces in Figure $4 \mathrm{~A}, \mathrm{C})$. We expect the $\mathrm{Fe} \mathrm{L}_{2,3}$-edges to respond on a time scale well below $1 \mathrm{ps}$, similar to the transient changes observed in optical spectroscopy measurements. ${ }^{24,34}$ Thus, the rise-time of the transients shown in Figure 4A,C is determined by the $70 \mathrm{ps} x$-ray probe pulses. We model the data with an instantaneous step-like molecular response convolved with a Gaussian instrument response of 70 ps FWHM such that $\Delta \mathrm{A}(\mathrm{E}, \mathrm{t})=\Delta \mathrm{A}_{0}(\mathrm{E}) / 2 \cdot[\operatorname{Erf}(\mathrm{t})+1]$. The fits of this model to the data (Figure 4A,C, solid lines) show good agreement, demonstrating that indeed the differential changes in the Ledge spectra, and hence the evolution of the Fe valence electronic structure, occur within the temporal resolution of the experiment. This is consistent with expectations based on the ultrafast spectroscopic studies described previously. ${ }^{23-26}$

Figure 5A shows the absorption spectra of the low-spin ${ }^{1} \mathrm{~A}_{1}$ state and the reconstructed high-spin ${ }^{5} \mathrm{~T}_{2}$ state at $90 \mathrm{ps}$ delay that results from the photo-induced metal-to-ligand charge transfer. The absorption spectrum of the low-spin state is in itself noteworthy as it is one of the few soft x-ray absorption spectra of solvated molecules. ${ }^{46,47}$ Recording absorption spectra in transmission circumvents the need for postexperimental corrections as is sometimes necessary in fluorescence and electron yield spectroscopy modes. ${ }^{48,49}$ In addition, transmission experiments are not affected by potential distortions of the spectral shape due to energy dependent yield channels, which can significantly affect fluorescence yield spectra. ${ }^{31}$ Transmission spectroscopy also serves to quantify absolute $x$-ray absorption cross sections and from our measurements we calculate a peak absorption crosssection at $708.5 \mathrm{eV}$ of $(1.0 \pm 0.3) \cdot 10^{-17} \mathrm{~cm}^{2}$. The high-spin absorption spectrum $A_{H S}$ is determined from the differential measurements $(\triangle \mathrm{A})$ in Figure $4 \mathrm{~B}$, using a photolysis fraction of $\mathrm{r}=0.18 \pm 0.02$ (deduced from experimental parameters) according to $A_{H S}(E)=A_{L S}(E)+\Delta A(E) / r$ with E being the energy of the $\mathrm{x}$-ray photons. Upon 560-nm excitation, the $\mathrm{L}_{3}$ edge of the high-spin state shows a pronounced red-shift by $1.7 \mathrm{eV}$ with some reshaping and an increased linewidth. The peak absorption is reduced while the total absorption in- 


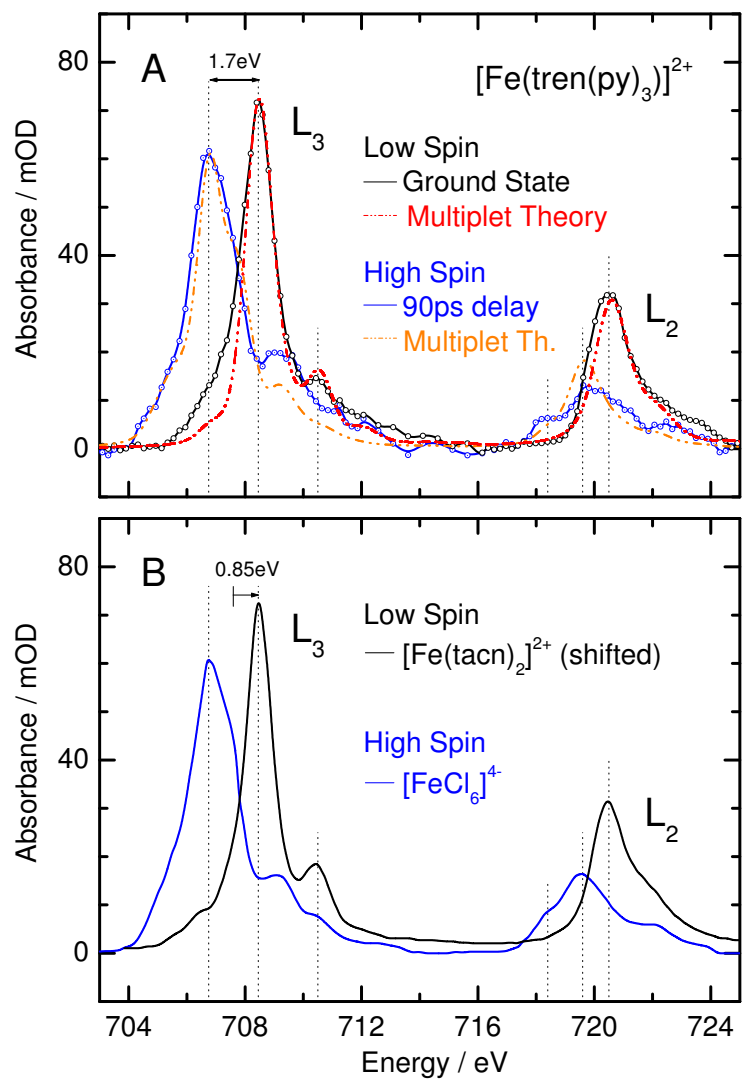

Figure 5. X-ray absorption spectra of various FeII complexes. (A) L-edge spectra of the low-spin ground state and photoinitiated transient high-spin state at 90 ps delay, reconstructed from the differential absorption spectrum in Figure $4 \mathrm{~B}$ by estimating that $(18 \pm 2) \%$ of the molecules were excited (black and blue open circles with spline fit, respectively) and charge transfer multiplet calculations (black and blue dotted lines). (B) Rescaled L-edge spectra of three crystalline compounds: $\left[\mathrm{Fe}(\operatorname{tacn})_{2}\right]^{2+}$ and $\left[\mathrm{FeCl}_{6}\right]^{4^{-}}$(solid dark red and blue line, respectively, both from ref 50 measured with an energy resolution of $0.2 \mathrm{eV}$ ) and $\left[\mathrm{Fe}(\operatorname{tpp})(\mathrm{ImH})_{2}\right]^{2+}$ (dash-dotted black line, taken from ref 51 measured with an energy resolution of $0.15 \mathrm{eV}$ ). See text for further details.

creases by $20 \%$. The $\mathrm{L}_{2}$-edge exhibits a shift of $\sim 1 \mathrm{eV}$ to lower energies upon laser excitation and the absorption crosssection reduces by nearly a factor of 2 . A distinct absorption increase below the low-spin $\mathrm{L}_{2}$-edge is visible at $718.5 \mathrm{eV}$.

Analysis of L-edge Features. Analysis and interpretation of these transient changes in the L-edge spectra provide new insights into the evolution of the $3 d$-orbitals in $\mathrm{Fe}^{\mathrm{II}}$ complexes accompanying the low-spin to high-spin conversion. However, in this spectral regime one must properly account for many-body effects among the increased number of valence electrons and between these valence electrons and the $\mathrm{Fe}-2 p$ core hole (generated by the $\mathrm{x}$-ray probe process during which the absorbed $\mathrm{x}$-ray photon promotes an inner-shell $\mathrm{Fe}$ $2 p$ electron into an empty $\mathrm{Fe}-3 d$ valence orbital). These many-body effects lead to spectroscopic multiplet structures that influence the shape of metal L and M-edges ${ }^{45}$ and preclude simple qualitative interpretations. In the following we develop a detailed understanding of the evolving electronic structure of the spin-crossover complex $\left[\mathrm{Fe}\left(\operatorname{tren}(\mathrm{py})_{3}\right)\right]^{2+}$ by (i) comparing our results to static investigations of crystalline iron complexes ${ }^{50,51}$ and (ii) employing charge transfer multiplet calculations. ${ }^{32}$ Our analysis leads to a detailed picture of valence charge delocalization (ligand charge delocalized into the metal, and metal charge delocalized into the ligand), quantification of the changes in ligand-field splitting of the Fe-3d $e_{\mathrm{g}}$ and $\mathrm{t}_{2 \mathrm{~g}}$ levels and their respective covalency, and shows that the influence of the ligand $\pi$-electron system is significantly reduced in the transient high-spin state.
Figure 5 presents a comparison of our results in panel A with static measurements of model crystalline iron complexes $^{50,51}$ in panel B: $\left[\mathrm{Fe}(\operatorname{tacn})_{2}\right]^{2+}$ (where tacn is 1,3,7triazacyclononane, a low-spin $\mathrm{Fe}^{1 \mathrm{II}}$ complex) and $\left[\mathrm{FeCl}_{6}\right]^{4-}$ (a high-spin $\mathrm{Fe}^{\mathrm{II}}$ complex). The model compounds exhibit spectra that are remarkably similar to those of the low-spin and photo-excited high-spin $\left[\mathrm{Fe}\left(\operatorname{tren}(\mathrm{py})_{3}\right)\right]^{2+}$ in Figure $5 \mathrm{~A}$, respectively. We will first focus on the comparison of the lowspin L-edges of $\left[\mathrm{Fe}\left(\operatorname{tren}(\mathrm{py})_{3}\right)\right]^{2+}$ with those of $\left[\mathrm{Fe}(\operatorname{tacn})_{2}\right]^{2+}$. Both compounds have comparable local ligand-field symmetries (strictly speaking $C_{3}$ but reasonably approximated as $O_{h}$ ) and consequently exhibit similar L-edge shapes, but the absence of $\pi$-type orbitals in $\left[\mathrm{Fe}(\operatorname{tacn})_{2}\right]^{2+}$ provides a useful contrast to $\left[\mathrm{Fe}\left(\operatorname{tren}(\mathrm{py})_{3}\right)\right]^{2+}$. In a recent study, Hocking and coworkers highlighted the influence of $\pi$-backbonding on the iron $\mathrm{L}_{2,3}$-edges ${ }^{51}$ in which the porphyrin-containing compound $\left[\mathrm{Fe}(\mathrm{tpp})(\mathrm{ImH})_{2}\right]$ (where tpp is tetraphenylporphyrin and $\mathrm{ImH}$ is imidazole) was compared with $\left[\mathrm{Fe}(\operatorname{tacn})_{2}\right]^{2+}$. The presence of the (electron-accepting) $\pi$-system of the porphyrin leads to a delocalization of the $\mathrm{Fe}-3 d$ electrons into the porphyrin ligand, resulting in $\mathrm{L}_{2,3}$-absorption edges at higher energies than those of $\left[\mathrm{Fe}(\operatorname{tacn})_{2}\right]^{2+}$ (Figure 5B). Notably, the $\mathrm{L}_{2,3}$-absorption peaks of the iron porphyrin coincide with the low-spin spectrum of our $\left[\mathrm{Fe}\left(\operatorname{tren}(\mathrm{py})_{3}\right)\right]^{2+}$, suggesting that $\pi$ backbonding is contributing significantly to the bonding in $\left[\mathrm{Fe}\left(\operatorname{tren}(\mathrm{py})_{3}\right)\right]^{2+}$. In other words, the energy shift between the spectra of $\left[\mathrm{Fe}\left(\operatorname{tren}(\mathrm{py})_{3}\right)\right]^{2+}$ and $\left[\mathrm{Fe}(\operatorname{tacn})_{2}\right]^{2+}$ in their ${ }^{1} \mathrm{~A}_{1}$ ground states provides a striking illustration of the effect of Fe-3 $d$ electron delocalization into the $\pi$-system of the $\operatorname{tren}(\mathrm{py})_{3}$ ligand.

Complementary to the comparison of the two low-spin spectra, the L-edges of the transiently formed ${ }^{5} \mathrm{~T}_{2}$ excited state of $\left[\mathrm{Fe}\left(\operatorname{tren}(\mathrm{py})_{3}\right)\right]^{2+}$ at $90 \mathrm{ps}$ time delay and the ${ }^{5} \mathrm{~T}_{2}$ ground state of $\left[\mathrm{FeCl}_{6}\right]^{4-}$ in Figure $5 \mathrm{~A}$ and $\mathrm{B}$ (blue solid lines) also show remarkable similarity and identical spectral positions. Although $\left[\mathrm{FeCl}_{6}\right]^{4-}$ has $\mathrm{t}_{2 \mathrm{~g}}$-symmetry ligand-group valence orbitals, they derive from the filled $3 p$ orbitals of the $\mathrm{Cl}^{-}$ ligands: as such, $\mathrm{Cl}^{-}$can only serve as a $\pi$-donor (in contrast to the $\pi$-acceptor character of the pyridyl groups in $\left.\operatorname{tren}(p y)_{3}\right)$. In this circumstance, the $t_{2 g}$ orbitals of Fe are formally $\pi$ antibonding, predominantly $3 d$-orbital in character, and essentially localized on the iron center. The strong similarity of both the high-spin L-edges in Figure 5A and B therefore allows us to conclude that $3 d$ electron delocalization is dramatically reduced in the photo-initiated transient high-spin state of $\left[\mathrm{Fe}\left(\operatorname{tren}(\mathrm{py})_{3}\right)\right]^{2+}$. This is due in large part to the increased metal-ligand bond distance in the high-spin state (which in turn causes an order-of-magnitude reduction in the oscillator strength of the ${ }^{5} \mathrm{~T}_{2} \rightarrow{ }^{5}$ MLCT transition in highspin species). ${ }^{24,34}$ An important conclusion from this comparison is that the photo-induced changes in the $\mathrm{Fe} \mathrm{L}$ absorption spectra in Figure 5A are a consequence not only of changes in $3 d$-orbital occupancy and a smaller ligand field, but are also a consequence of suppressed $3 d$-electron delocalization. In particular, the large shift of the $\mathrm{L}_{3}$-edge by $1.7 \mathrm{eV}$ reflects the strongly reduced influence of the ligand $\pi$ electron system on the metal-3d orbitals. Given that the overall absorption remains constant (see above), these competing effects roughly offset each other in the low-spin to high-spin conversion of $\left[\mathrm{Fe}\left(\operatorname{tren}(\mathrm{py})_{3}\right)\right]^{2+}$.

Charge transfer multiplet calculations ${ }^{32}$ for the low-spin and high-spin L-edges of the $\left[\mathrm{Fe}\left(\operatorname{tren}(\mathrm{py})_{3}\right)\right]$ spin-crossover compound are plotted in Figure $5 \mathrm{~A}$ as dash-dotted lines (red and orange, respectively). The multiplet calculations provide a detailed picture that takes into account important delocalization of ligand- $2 p$ electron density into unoccupied metal- $3 d$ orbitals ( $\sigma$-donation) and $\mathrm{Fe}-3 d$ delocalization into unoccupied ligand- $2 p$ orbitals ( $\pi$-backbonding) ${ }^{52}$ In particular, our simulations indicate an initial ligand-field splitting of $\Delta \mathrm{E}_{\mathrm{LS}}=$ $1.7 \mathrm{eV}$ in the low-spin state that reduces to $\Delta \mathrm{E}_{\mathrm{HS}}=0.6 \mathrm{eV}$ in 
the high-spin state. These values are $\sim 20 \%$ lower than those typically derived from optical spectroscopy, and are a consequence of $\mathrm{Fe}-2 p$ core hole effects ${ }^{53}$ inherent to the measurement process (the ligand-field splitting without core hole effects will be $\sim 2 \mathrm{eV}$ and $\sim 1 \mathrm{eV}$ for the low and high-spin state, respectively). For a ligand-field splitting $\Delta \mathrm{E}>1.6 \mathrm{eV}$, iron complexes usually favor the low-spin state ${ }^{16}$ with all six Fe$3 d$ electrons paired in the $\mathrm{t}_{2 \mathrm{~g}}$ orbitals: in this configuration, the $\mathrm{L}_{2,3}$-transitions exclusively probe the empty $\mathrm{e}_{\mathrm{g}}$ orbitals. As the system evolves subsequent to photoexcitation, the ligand field strength is attenuated concomitant with evolution along the ${ }^{5} \mathrm{~T}_{2}$ potential surface. The nominally higher energy cost of $\mathrm{e}_{\mathrm{g}}$ occupancy is more than compensated by the overall decrease in spin-pairing energy, hence the establishment of a $\left(t_{2 g}\right)^{4}\left(e_{g}\right)^{2}$ configuration for the ${ }^{5} T_{2}$ term. Some discrepancies between the $\mathrm{L}_{2}$-edges of the experimental high-spin complex and the corresponding multiplet calculation in Figure 5A hint at reduced spin-orbit coupling between $3 d$-electrons in the high-spin state of $\left[\mathrm{Fe}\left(\operatorname{tren}(\mathrm{py})_{3}\right)\right]^{2+}$ : preliminary multiplet calculations with suppressed spin-orbit coupling between $3 d$ electrons significantly shift absorption from the peak of the $\mathrm{L}_{2}$-edge at $719.6 \mathrm{eV}$ to the pre-edge feature at $718.4 \mathrm{eV}$ while increasing the height of the $\mathrm{L}_{3}$-post-edge at $709.2 \mathrm{eV}$.

The photo-initiated spin crossover is accompanied by weaker metal-ligand interactions and increased metal $3 d$ character of the central iron atom. According to the multiplet calculations, this is manifest by a significant reduction in covalent mixing of the Fe $3 d$-orbitals with neighboring $\mathrm{N}-2 p$ orbitals. Covalency mediates charge delocalization between the metal ion and its ligands and influences the orbital occupancy and hence, the effective number of empty orbitals which determines the absorption cross-section. While $\sigma$ donation increases the occupancy of the $\mathrm{e}_{\mathrm{g}}$ orbitals (due to their orientation along the $\mathrm{Fe}-\mathrm{N}$ bond axes), $\pi$-backbonding decreases the occupancy of $t_{2 g}$ orbitals. As discussed above, the integral over the differential spectrum at 90 ps delay in Figure 4B vanishes within the accuracy of the measurement, suggesting a comparable $\mathrm{Fe}-3 d$ orbital occupancy in both the low-spin and high-spin states. Consequently, the aforementioned reduction in $\pi$-backbonding is compensated by the simultaneous decrease in $\sigma$-donation.

Finally, we compare our spectral results to the difference of two L-edge spectra of the crystalline spin cross-over compound $\mathrm{Fe}^{\mathrm{II}}(\text { phen })_{2}(\mathrm{NCS})_{2}$, plotted in Figure 4B (grey line). The difference spectrum is derived from static measurements of the low-spin and high-spin $\mathrm{L}_{23}$-edges by Briois and coworkers. ${ }^{54}$ This crystalline compound exhibits a thermally driven phase transition from the low-spin to the high-spin state at a critical temperature of $T_{c}=176 \mathrm{~K}$ and shows similarity to $\left[\mathrm{Fe}\left(\operatorname{tren}(\mathrm{py})_{3}\right)\right]^{2+}$ with respect to the spectral behavior at the $\mathrm{L}_{2,3}$-edges and the iron $\mathrm{K}$-edge. The shift of the $\mathrm{L}_{3}$-edge to lower energy is smaller which could signal stronger metalligand interactions in the high-spin state compared to $\left[\mathrm{Fe}\left(\operatorname{tren}(\mathrm{py})_{3}\right)\right]^{2+}$. Brois and co-workers have not reported relative absorption cross sections for the high-spin and lowspin L-edge measurements but we have calculated the grey difference spectrum in Figure $4 \mathrm{~B}$ according to $\Delta \mathrm{A}=0.9 \cdot \mathrm{A}_{\mathrm{HS}}$ - $A_{L S}$ which yields the same change in total absorption crosssection as $\left[\mathrm{Fe}\left(\operatorname{tren}(\mathrm{py})_{3}\right)\right]^{2+}$. This rescaled difference also shows the best match with line-shape features of the difference spectrum derived from similar work on $\mathrm{Fe}^{\mathrm{II}}$ (phen $)_{2}(\mathrm{NCS})_{2}$ by Lee and co-workers ${ }^{55}$.

Solvent Effects. One of the more significant aspects of this study is the fact that the measurements were carried out in fluid solution: it is therefore reasonable to consider the extent to which the solvent could be influencing the data we have presented. Solvation dynamics consist of distinct processes that can be differentiated with respect to the time scale on which they occur. In the sub-picosecond domain where the formation of the ${ }^{5} \mathrm{~T}_{2}$ state occurs, ${ }^{34}$ inertial dynamics are dominant (the inertial response time for the solvent $\mathrm{CH}_{3} \mathrm{CN}$ is $\sim 60 \mathrm{fs}) .{ }^{56,57}$ While such sub-picosecond dynamics have been shown to influence the excited-state evolution of chargetransfer states, ${ }^{58}$ the $\sim 50$ ps resolution of our experiment infers that there should be negligible contribution from such processes to the results we have described herein.

Rotational correlation times for solvents such as $\mathrm{CH}_{3} \mathrm{CN}$ are on the order of tens of picoseconds: this is still faster than the time scale of our measurements but could in principle affect our observations. In this regard, the nature of the excited state we are probing is relevant. Ligand-field states, such as the ${ }^{5} \mathrm{~T}_{2}$ state probed in $\left[\mathrm{Fe}\left(\operatorname{tren}(\mathrm{py})_{3}\right)\right]^{2+}$, are by their very nature metal-localized. Although our data clearly shows evidence of delocalization involving the coordinated pyridyl rings, the metal-ligand interactions in first-row transition metal complexes are still predominantly ionic in nature. The ligand framework - particularly in compounds such as $\left[\mathrm{Fe}\left(\operatorname{tren}(\mathrm{py})_{3}\right)\right]^{2+}$ - imparts both a steric and dielectric shield of the metal center from the solvent. The absence of significant solvatochromism associated with the ligand-field absorption spectra of most metal complexes serves to underscore this point. Based on these considerations, we don't believe that the solvent is having a significant impact on the results we have described.

In contrast to the dynamics of ligand-field states, solvent effects may indeed be important in the transition from the short-lived charge-transfer (CT) states ${ }^{24,35}$ to the ligand-field manifold (LFM) of first-row metal complexes. Improved time resolution in solution-phase L-edge measurements could provide crucial insights into the reaction mechanism associated with $\mathrm{CT} \rightarrow \mathrm{LFM}$ transitions (and subsequent transitions between LFM states). Transient L-edge spectroscopy with time resolution equivalent to the lifetime of the $\mathrm{CT}$ state would also report on symmetry-specific charge delocalization in the CT state and the nature of transiently populated LFM states (for instance, transient triplet state formation if existent). Such studies would also aid in exploring first-row metal complexes in certain solar energy conversion strategies: ${ }^{37}$. Efforts to study the solvent influence in a systematic manner are currently underway. Beyond the solvent influence on CT states, molecular mechanisms in many important systems such as enzymatic reactions in metalloproteins and ligand substitution reactions involving metal-inorganic compounds exhibit transient states that can only be studied in solution with high time-resolution. So far as we are aware, our experiment is the first to offer a direct comparison of initial and final state L-edge spectra in the same solvated complex.

\section{Conclusions}

This work reports the first time-resolved soft x-ray spectroscopy of electron and spin dynamics in solvated molecules. We studied a $\mathrm{Fe}^{\mathrm{II}}$ spin-crossover complex by probing photo-initiated transient absorption changes of the iron $\mathrm{L}_{2,3^{-}}$ edges. The observed large shift of the $\mathrm{L}_{2,3}$ absorption edges to lower energies upon spin crossover is caused not only by a reduction in ligand-field splitting and changes in $\mathrm{Fe}-3 d \mathrm{e}_{\mathrm{g}}$ and $t_{2 g}$ orbital occupancy but necessitates a strongly reduced interaction between the $\mathrm{Fe}-3 d$ electron and the $\pi$-electron system of the ligands. Upon spin crossover, the resulting ligand cage dilation attenuates metal-ligand interactions thereby reducing the ligand-field splitting of the Fe-3d orbitals by $\sim 1$ $\mathrm{eV}$ while increasing the ionic character of the system without changing the effective electronic occupancy of these orbitals.

The time-resolved L-edge spectroscopic study presented here constitutes a prototype experiment that captures the transients changes in valence-orbital structure in a solvated transition-metal system in a very detailed manner. This new technique is generally well suited to examine ligand-metal interactions, offering future opportunities to investigate important classes of transition metal compounds such as protein co- 
factors and mixed-valence compounds, and more generally to study photo-initiated liquid-phase chemistry over a wide range of time-scales from sub-femtoseconds to milliseconds.

Acknowledgments. This work was supported by the Director, Office of Science, Office of Basic Energy Sciences, the Chemical Sciences, Geosciences, and Biosciences Division under the Department of Energy Contract No. DE-AC02-05CH11231 (N.H. and R.W.S.) and Grant No. DE-FG0201ER15282 (J.K.M.), as well as Grant No. R-01-2008-000-20717-0 of the Basic Research Program of the Korea Science and Engineering Foundation, Republic of Korea (T.K.K.) and Grant No. 700.56 .443 of the Netherlands National Science Foundation (NWO) VICI program (F.d.G.).

Legal Disclaimer. This document was prepared as an account of work sponsored by the United States Government. While this document is believed to contain correct information, neither the United States Government nor any agency thereof, nor The Regents of the University of California, nor any of their employees, makes any warranty, express or implied, or assumes any legal responsibility for the accuracy, completeness, or usefulness of any information, apparatus, product, or process disclosed, or represents that its use would not infringe privately owned rights. Reference herein to any specific commercial product, process, or service by its trade name, trademark, manufacturer, or otherwise, does not necessarily constitute or imply its endorsement, recommendation, or favoring by the United States Government or any agency thereof, or The Regents of the University of California. The views and opinions of authors expressed herein do not necessarily state or reflect those of the United States Government or any agency thereof or The Regents of the University of California..

\section{References.}

1. Maroncelli, M.; Fleming, G. R. J. Chem. Phys. 1987, 86, 6221-6239.

2. Schoenlein, R. W.; Peteanu, L. A.; Mathies, R. A.; Shank, C. V. Science 1991, 254, 412-415.

3. Fidder, H.; Terpstra, J.; Wiersma, D. A. J. Chem. Phys. 1991, 94, 68956907

4. Zewail, A. H. J. Phys. Chem. 1993, 97, 12427-12446.

5. Hamm, P.; Lim, M.; Hochstrasser, R. M. J. Phys. Chem. B 1998, 102, 6123-6138.

6. Rini, M.; Magnes, B. Z.; Pines, E.; Nibbering, E. T. J. Science 2003, 301, 349-352.

7. McCusker, J. K. Acc. Chem. Res. 2003, 36, 876-887.

8. Chen, L. X.; Lee, P. L.; Gosztola, D. J.; Svec, W. A.; Wasielewski, M. R. J. Synchrotron Rad. 1999, 6, 403-405.

9. Cavalleri, A.; Rini, M.; Chong, H. H. W.; Fourmaux, S.; Glover, T. E.; Heimann, P. A.; Kieffer, J. C.; Schoenlein, R. W. Phys. Rev. Lett. 2005, 95, 067405 .

10. Lee, T.; Jiang, Y.; Rose-Petruck, C. G.; Benesch, F. J. Chem. Phys. 2005, 122, 084506.

11. Smolentsev, G.; Soldatov, A. V.; Chen, L. X. J. Phys. Chem. A 2008 $112,5363-5367$.

12. Bressler, C.; Milne, C.; Pham, V.-T.; ElNahhas, A.; van der Veen, R. M.; Gawelda, W.; Johnson, S.; Beaud, P.; Grolimund, D.; Kaiser, M.; Borca, C. N.; Ingold, G.; Abela, R.; Chergui, M. Science 2009, 323, 489-492.

13. Wen, H.; Huse, N.; Schoenlein, R. W.; Lindenberg, A. M. J. Chem. Phys. 2009, 131, 234505.

14. Stöhr, J. NEXAFS Spectroscopy, volume 25 of Springer Series in Surface Sciences. Springer-Verlag, Berlin 1992

15. Holm, R. H.; Kennepohl, P.; Solomon, E. I. Chem. Rev. 1996, 96, 2239.

16. Gütlich, P.; Goodwin, H. A., Eds. Topics in Current Chemistry: Spin Crossover in Transition Metal Compounds, volume 233-235. Springer, Berlin / Heidelberg 2004.

17. Decurtins, S.; Gütlich, P.; Köhler, C.; Spiering, H.; Hauser, A. Chem. Phys. Lett. 1984, 105, 1-4.

18. Ferrere, S.; Gregg, B. A. J. Am. Chem. Soc. 1998, 120, 843-844.

19. Létard, J.-F. J. Mater. Chem. 2006, 16, 2550-2559. , and references therein.

20. Schoenlein, R. W.; Chattopadhyay, S.; Chong, H. H. W.; Glover, T. E.; Heimann, P. A.; Shank, C. V.; Zholents, A. A.; Zolotorev, M. S. Science 2000, 287, 2237-2240

21. Heimann, P. A.; Padmore, H. A.; Schoenlein, R. W. AIP Conf. Proc., May 2004, 705(1), 1407-1410.
22. Heimann, P. A.; Glover, T. E.; Plate, D.; Lee, H. J.; Brown, V. C.; Padmore, H. A.; Schoenlein, R. W. AIP Conf. Proc. 2007, 879, 1195.

23. Attwood, D. T. Soft X-rays and Extreme Ultraviolet Radiation: Principles and Applications. Cambridge University Press 1999.

24. Monat, J. E.; McCusker, J. K. J. Am. Chem. Soc. 2000, 122, 4092-4097.

25. Yang, B. X.; Kirz, J. Phys. Rev. B 1987, 36, 1361-1364.

26. Wernet, P.; Gavrila, G.; Godehusen, K.; Weniger, C.; Nibbering, E.; Elsaesser, T.; Eberhardt, W. Appl. Phys. A: Mater. Sci. Process. 2008, $92,511-516$.

27. Huse, N.; Wen, H.; Nordlund, D.; Szilagyi, E.; Daranciang, D.; Miller, T. A.; Nilsson, A.; Schoenlein, R. W.; Lindenberg, A. M. Phys. Chem. Chem. Phys. 2009, 11, 3951-3957.

28. de Groot, F. M. F.; de Smit, E.; van Schooneveld, M. M.; Aramburo, L. R.; Weckhuysen, B. M. Chem. Phys. Chem. 2010, 11, 951 - 962.

29. Kraemer, D.; Cowan, M. L.; Paarmann, A.; Huse, N.; Nibbering, E. T. J.; Elsaesser, T.; Miller, R. J. D. Proc. Nat. Acad. Sci. 2008, 105, 437-442.

30. Huse, N.; Khalil, M.; Kim, T. K.; Smeigh, A. L.; Jamula, L.; McCusker, J. K.; Schoenlein, R. W. J. Phys: Conf. Ser. 2009, 148, 012043.

31. Thole, B. T.; Cowan, R. D.; Sawatzky, G. A.; Fink, J.; Fuggle, J. C. Phys. Rev. B 1985, 31, 6856-6858.

32. de Groot, F.; Kotani, A. Core Level Spectroscopy of Solids. Advances in Condensed Matter Science. Taylor \& Francis 2008.

33. Khalil, M.; Marcus, M. A.; Smeigh, A. L.; McCusker, J. K.; Chong, H. H. W.; Schoenlein, R. W. J. Phys. Chem. A 2006, 110, 38-44.

34. Smeigh, A. L.; Creelman, M.; Mathies, R. A.; McCusker, J. K. J. Am. Chem. Soc. 2008, 130, 14105-14107.

35. Gawelda, W.; Cannizzo, A.; Pham, V.-T.; van Mourik, F.; Bressler, C.; Chergui, M. J. Am. Chem. Soc. 2007, 129, 8199-8206.

36. Consani, C.; Prémont-Schwarz, M.; ElNahhas, A.; Bressler, C.; van Mourik, F.; Cannizzo, A.; Chergui, M. Angew. Chem. Int. Ed. 2009, 48, $7184-7187$.

37. Smeigh, A. L.; Brown, A.; McCusker, J. K. manuscript in preparation.

38. Gawelda, W.; Pham, V.-T.; Benfatto, M.; Zaushitsyn, Y.; Kaiser, M.; Grolimund, D.; Johnson, S. L.; Abela, R.; Hauser, A.; Bressler, C.; Chergui, M. Phys. Rev. Lett. 2007, 98, 057401.

39. Nozawa, S.; Sato, T.; Chollet, M.; Ichiyanagi, K.; Tomita, A.; Fujii, H.; Adachi, S.-i.; Koshihara, S.-y. J. Am. Chem. Soc. 2010, 132, 61-63.

40. The lifetime of the ${ }^{5} \mathrm{~T}_{2}$ state of $\left[\mathrm{Fe}\left(\operatorname{tren}(\mathrm{py})_{3}\right)\right]^{2+}$ in fluid solution at room temperature is $(60 \pm 5) \mathrm{ns}$

41. de Groot, F. M. F.; Fuggle, J. C.; Thole, B. T.; Sawatzky, G. A. Phys. Rev. B 1990, 41, 928-937.

42. de Groot, F. M. F.; Fuggle, J. C.; Thole, B. T.; Sawatzky, G. A. Phys. Rev. B 1990, 42, 5459-5468.

43. de Groot, F. M. F.; Hu, Z. W.; Lopez, M. F.; Kaindl, G.; Guillot, F.; Tronc, M. J. Chem. Phys. 1994, 101, 6570-6576.

44. Thole, B. T.; van der Laan, G. Phys. Rev. B, Aug 1988, 38, 3158-3171.

45. de Groot, F. Coord. Chem. Rev. 2005, 249, 31-63.

46. Bonhommeau, S.; Ottosson, N.; Pokapanich, W.; Svensson, S.; Eberhardt, W.; Björneholm, O.; Aziz, E. F. J. Phys. Chem. A 2008, 112, 12571-12574.

47. Aziz, E. F.; Ottosson, N.; Bonhommeau, S.; Bergmann, N.; Eberhardt, W.; Chergui, M. Phys. Rev. Lett. 2009, 102, 068103.

48. de Groot, F. M. F.; Arrio, M. A.; Sainctavit, P.; Cartier, C.; Chen, C. T. Solid State Communications 1994, 92, 991-995.

49. Nakajima, R.; Stöhr, J.; Idzerda, Y. U. Phys. Rev. B 1999, 59, 64216429 .

50. Wasinger, E. C.; de Groot, F. M. F.; Hedman, B.; Hodgson, K. O.; Solomon, E. I. J. Am. Chem. Soc. 2003, 125, 12894-12906

51. Hocking, R. K.; Wasinger, E. C.; Yan, Y. L.; deGroot, F. M. F.; Walker, F. A.; Hodgson, K. O.; Hedman, B.; Solomon, E. I. J. Am. Chem. Soc. 2007, 129, 113-125.

52. Hocking, R. K.; Wasinger, E. C.; de Groot, F. M. F.; Hodgson, K. O.; Hedman, B.; Solomon, E. I. J. Am. Chem. Soc. 2006, 128, 10442-10451.

53. Cramer, S. P.; de Groot, F. M. F.; Ma, Y.; Chen, C. T.; Sette, F.; Kipke, C. A.; Eichhorn, D. M.; Chan, M. K.; Armstrong, W. H.; Libby, E.; Christou, G.; Brooker, S.; McKee, V.; Mullins, O. C.; Fuggle, J. C. J. Am. Chem. Soc. 1991, 113, 7937-7940.

54. Briois, V.; dit Moulin, C. C.; Sainctavit, P.; Brouder, C.; Flank, A.-M. J. Am. Chem. Soc. 1995, 117, 1019-1026.

55. Lee, J.-J.; Sheu, H.-s.; Lee, C.-R.; Chen, J.-M.; Lee, J.-F.; Wang, C.-C.; Huang, C.-H.; Wang, Y. J. Am. Chem. Soc. 2000, 122, 5742-5747.

56. Stratt, R. M.; Maroncelli, M. J. Phys. Chem. 1996, 100, 12981-12996.

57. Passino, S. A.; Nagasawa, Y.; Fleming, G. R. J. Chem. Phys. 1997, 107, 6094-6108

58. Yeh, A. T.; Shank, C. V.; McCusker, J. K. Science 2000, 289, 935-93 